\title{
In vitro reversion of activated primary human hepatic stellate cells
}

\author{
Adil El Taghdouini ${ }^{1}$, Mustapha Najimi ${ }^{2}$ Pau Sancho-Bru', Etienne Sokal ${ }^{2}$ and Leo A. van Grunsven ${ }^{1 *}$
}

\begin{abstract}
Background: Liver fibrosis is characterized by the excessive formation and accumulation of matrix proteins as a result of wound healing in the liver. A main event during fibrogenesis is the activation of the liver resident quiescent hepatic stellate cell (qHSC). Recent studies suggest that reversion of the activated HSC (aHSC) phenotype into a quiescent-like phenotype could be a major cellular mechanism underlying fibrosis regression in the liver, thereby offering new therapeutic perspectives for the treatment of liver fibrosis. Whether human HSCs have the ability to undergo a similar reversion in phenotype is currently unknown. The aim of the present study is to identify experimental conditions that can revert the in vitro activated phenotype of primary human HSCs and consequently to map the molecular events associated with this reversion process by gene expression profiling.
\end{abstract}

Results: We find that epidermal growth factor (EGF) and fibroblast growth factor 2 (FGF2) synergistically downregulate the expression of ACTA2 and LOX in primary human aHSCs. Their combination with oleic acid, palmitic acid, and retinol further potentiates a more quiescent-like phenotype as demonstrated by the abundant presence of retinyl ester-positive intra-cytoplasmic lipid droplets, low expression levels of activation markers, and a reduced basal as well as cytokine-stimulated proliferation and matrix metalloproteinase activity. Gene expression profiling experiments reveal that these in vitro reverted primary human HSCS (rHSCs) display an intermediary phenotype that is distinct from qHSCs and aHSCs. Interestingly, this intermediary phenotype is characterized by the increased expression of several previously identified signature genes of in vivo inactivated mouse HSCs such as CXCL1, CXCL2, and CTSS, suggesting also a potential role for these genes in promoting a quiescent-like phenotype in human HSCS.

Conclusions: We provide evidence for the ability of human primary aHSCs to revert in vitro to a transitional state through synergistic action of EGF, FGF2, dietary fatty acids and retinol, and provide a first phenotypic and genomic characterization of human in vitro rHSCs.

Keywords: Fibrosis, Hepatic stellate cells, Reversion, Quiescent, Inactivation, Gene expression profiling

\section{Background}

In response to injury, tissue can heal and restore its normal function through a controlled sequence of events known as wound healing. However, when the insult is of chronic nature, it can impair the wound healing response and subsequently develop into tissue fibrosis, a condition characterized by the excessive accumulation of matrix proteins and associated with severe morbidity and mortality [1]. Different cellular sources, including tissue-specific fibroblasts, bone

\footnotetext{
* Correspondence: Ivgrunsv@vub.ac.be

'Liver Cell Biology Lab, Vrije Universiteit Brussel (VUB), Laarbeeklaan 103, 1090 Brussels, Belgium

Full list of author information is available at the end of the article
}

marrow-derived progenitor cells, pericytes, and epithelial cells, have been suggested to give rise to myofibroblasts, the major source of extracellular matrix (ECM) components in the fibrotic organ [2]. In the liver, however, the resident hepatic stellate cell (HSC) has unambiguously been identified as the predominant source of myofibroblasts, irrespective of the underlying disease etiology [3].

In the normal liver, quiescent HSCs (qHSCs) reside in a virtual space (of Disse) between the hepatocytes and liver sinusoidal endothelial cells and are characterized by the abundance of cytoplasmic lipid droplets containing up to $80 \%$ of the total vitamin A body reserve [4]. Besides their well-known role in the regulation of retinoid and ECM 
homeostasis, there is evidence that HSCs can regulate the sinusoidal blood flow and stimulate angiogenesis $[5,6]$. Following chronic liver injury, qHSCs undergo a process of activation, during which they transdifferentiate into cells with a fibrogenic, myofibroblast-like phenotype characterized by the loss of vitamin A-containing lipid droplets, increased smooth muscle actin (ACTA2) expression, and an augmentation in ECM production and secretion [4, 7].

Initially, this activation process was considered to be unidirectional while the principal ability of the fibrotic liver to revert to a normal state upon cessation of injury [8] was mainly attributed to the clearance of activated HSCs (aHSCs) that undergo apoptosis $[9,10]$. However, different studies strongly imply that at least in rodents, the activated phenotype of HSCs can be modulated and reverted to a quiescent-like state, both in vitro $[11,12]$ and in vivo $[13,14]$. The identification of aHSCs as the main source of ECM in the fibrotic liver in conjunction with recent work showing that HSC inactivation could drive fibrosis regression opens new therapeutic perspectives. Indeed, identifying and understanding the molecular events governing the reversion of an aHSC will be helpful in efficiently targeting liver fibrosis. Although convincingly shown in rodent HSCs, inactivating conditions for human primary HSCs have barely been investigated. In the present study, we aimed at identifying experimental conditions that can induce a reversion of the activated human HSC phenotype and at mapping the molecular events associated with this process.

We find that epidermal growth factor (EGF) and fibroblast growth factor 2 (FGF2) act synergistically to downregulate the expression of $A C T A 2$ and lysyl oxidase (LOX) in primary human HSCs. The combined effect is further potentiated by dietary fatty acids and retinol to reach levels of expression that are similar to those measured in freshly isolated, non-cultured human qHSCs. Further, this cocktail induces a quiescent-like phenotype as demonstrated by the acquisition of retinyl ester-containing cytoplasmic lipid droplets and a low proliferation rate. Gene expression profiling of human primary qHSCs and in vitro aHSCs and reverted HSCs (rHSCs) reveals many additional profibrogenic genes to be downregulated in rHSCs. However, despite their phenotype, the overall expression profile of rHSCs is not similar to that of qHSCs and differs from that of aHSCs, but shows increased expression of previously identified in vivo inactivated mouse HSC (iaHSC) signature genes $[13,15]$.

\section{Results}

EGF and FGF2 act synergistically with dietary fatty acids and retinol to revert the culture activated phenotype of human HSCs

Human HSCs are isolated by density gradient centrifugation from the non-parenchymal cell fraction of healthy liver donors (Table 1) and seeded on plastic culture dishes to induce activation (Fig. 1a, b). The activated status of the cells is assessed by their typical myofibroblastic phenotype, the strong increase in alpha smooth muscle actin $(\alpha-$ SMA) protein by immunocytochemistry (Fig. 1b) and collagen type 1 alpha 1 (COL1A1) and lysyl oxidase (LOX) expression by RT-qPCR (Fig. 1c). The aHSCs express high protein levels of platelet-derived growth factor receptor beta (PDGFR $\beta$ ), a membrane receptor associated with HSCs (Fig. 1d). Moreover, the cells stain positive for different markers previously shown to be expressed by HSCs, i.e., NCAM1, nestin, and desmin [16-19] (Fig. 1e).

We have tested different culture conditions for their ability to revert the activated phenotype of HSCs. These conditions include pharmacological agents previously shown to inhibit rodent HSC activation (i.e., TWS119, valproic acid (VPA), valinomycin, carbonyl cyanide- $p$-trifluoromethoxyphenylhydrazone (FCCP), tin-protoporphyrin IX dichloride (SnPP), bafilomycin (BFM), hydroxychloroquine (HCQ), 3methyladenine (3-MA)) (Additional file 1: Figure S1A), an adipogenic differentiation mixture (MDI) [12] (Additional file 1: Figure S1B), and a diverse pool of growth factors and cytokines (data not shown). While our experiments reveal that these agents merely induce cell death (Valinomycin, BFM, 3-MA) in human HSCs or show limited efficacy to inactivate the cells especially at the level of COL1A1 expression (TWS119, VPA, FCCP, SnPP, HCQ, MDI), they also reveal that FGF2 and EGF significantly downregulate the expression of $A C T A 2$ or $A C T A 2$ and $L O X$, respectively (Fig. 2a). We further find that the combined effect of EGF and FGF2 on the expression of ACTA2, COL1A1, and LOX expression is strongly potentiated by a mixture of dietary fatty acids (oleic acid (OA); palmitic acid (PA)) and retinol (R) to reach levels of expression that are similar to those measured in freshly isolated, non-cultured qHSCs (Fig. 2b), with decreased levels of ACTA2 and COL1A1 confirmed at the protein level (Fig. 2c). Of note, the mixture of dietary components alone has no significant effect on activation (Fig. 2a). HSCs reverted by this mix of growth factors and dietary components (further referred to as reverting medium (RM)) display a quiescent-like phenotype, characterized by a thinner cell body and the presence of intracytoplasmic lipid droplets (Fig. 3a). Although HSCs have strongly reduced expression levels of activation genes as

Table 1 Clinical characteristics of the HSC donors

\begin{tabular}{lllll}
\hline Donor & Healthy status & Age & Gender & Ischemia time \\
\hline L4 & Healthy & 12 years & Female & $16 \mathrm{~h} 30 \mathrm{~min}$ \\
L8 & Healthy & 1 day & Male & $4 \mathrm{~h} 40 \mathrm{~min}$ \\
L10 & Healthy & 7 months & Female & $5 \mathrm{~h} 20 \mathrm{~min}$ \\
L11 & Healthy & 7 days & Male & $4 \mathrm{~h} 25 \mathrm{~min}$ \\
L12 & FH & 13 years & Male & $1 \mathrm{~h} 30 \mathrm{~min}$ \\
\hline
\end{tabular}

FH familial hypercholesterolemia 


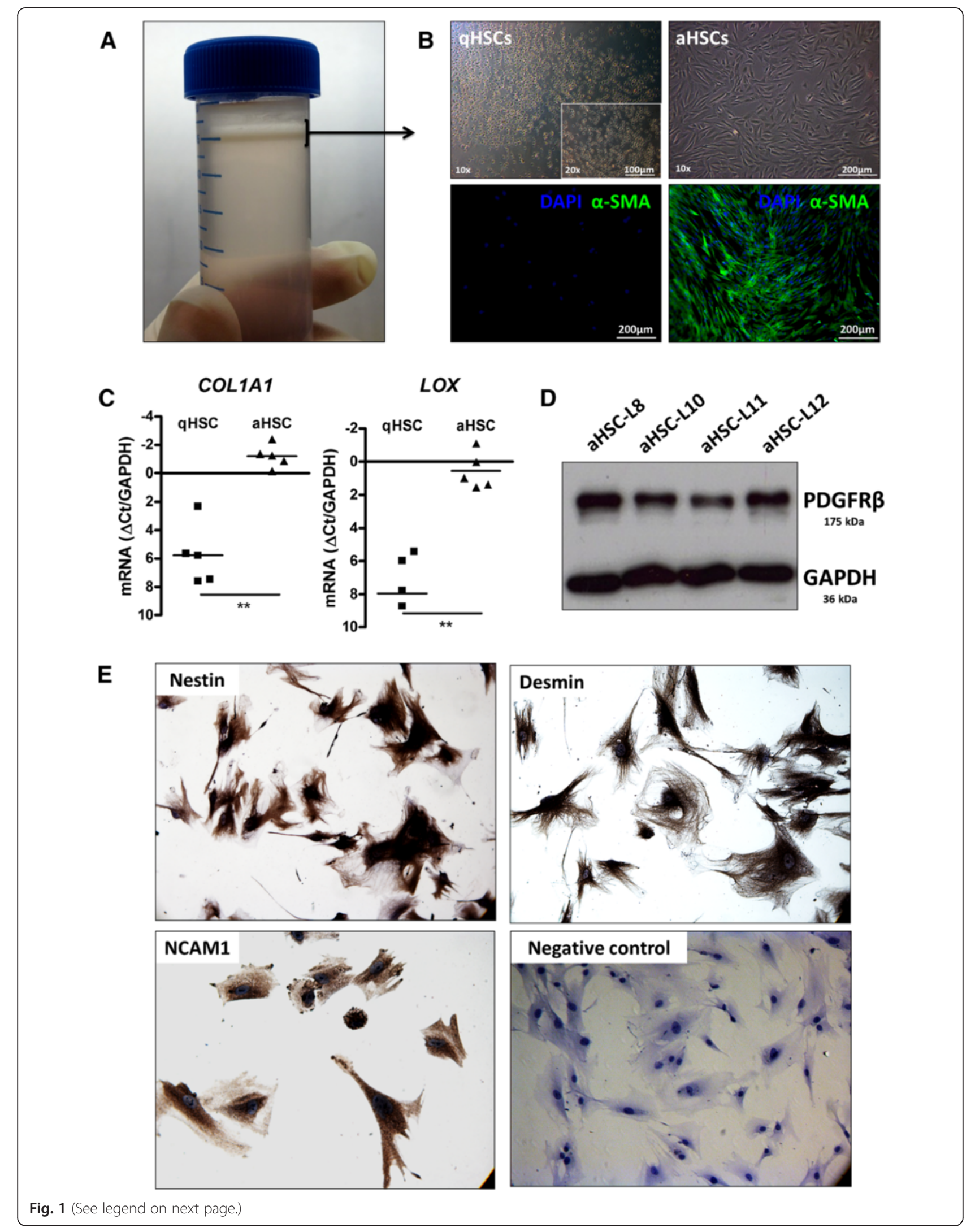


(See figure on previous page.)

Fig. 1 Isolation and culture activation of human primary HSCs. a Quiescent HSC-enriched cell layer (arrow) following an 8 \% Nycodenz density gradient centrifugation. $\mathbf{b}$ Light microscopic and a-SMA immunocytochemistry images of the freshly isolated, qHSC-enriched cell population and fully culture activated HSCs (passage 4). c Log2 mRNA expression levels of COL1A1 and LOX in freshly isolated, non-plated qHSCS and aHSCs from five different donors. In the graphs, ${ }^{* *} p<0.01$. d PDGFR $\beta$ and GAPDH protein levels in aHSCs from four donors. e Positive immunostainings for the neural markers NCAM1, nestin, and desmin

soon as 2 days after incubation with RM, intra-cytoplasmic lipid droplet accumulation is only observable after 4 days (Additional file 1: Figure S2). Therefore, functional analysis and gene expression profiling were performed on cells that were incubated with RM for at least 4 days.

In order to assess whether the in vitro reverted HSCs (rHSCs) have the molecular machinery both to metabolize and store vitamin A, a functional hallmark of qHSCs, we measured the retinyl ester auto-fluorescence at $328 \mathrm{~nm}$ by fluorescence-activated cell sorting (FACS). Approximately $70 \%$ of the aHSCs cultured in RM are $\mathrm{UV}^{+}$(Fig. 3b), and analysis of the rHSCs under UV light shows that the autofluorescent signal co-localizes with the intra-cytoplasmic lipid droplets, indicating that vitamin A is indeed metabolized and stored inside the lipid droplets (Fig. 3b). Although the majority of rHSCs are highly $\mathrm{UV}^{+}$, some of
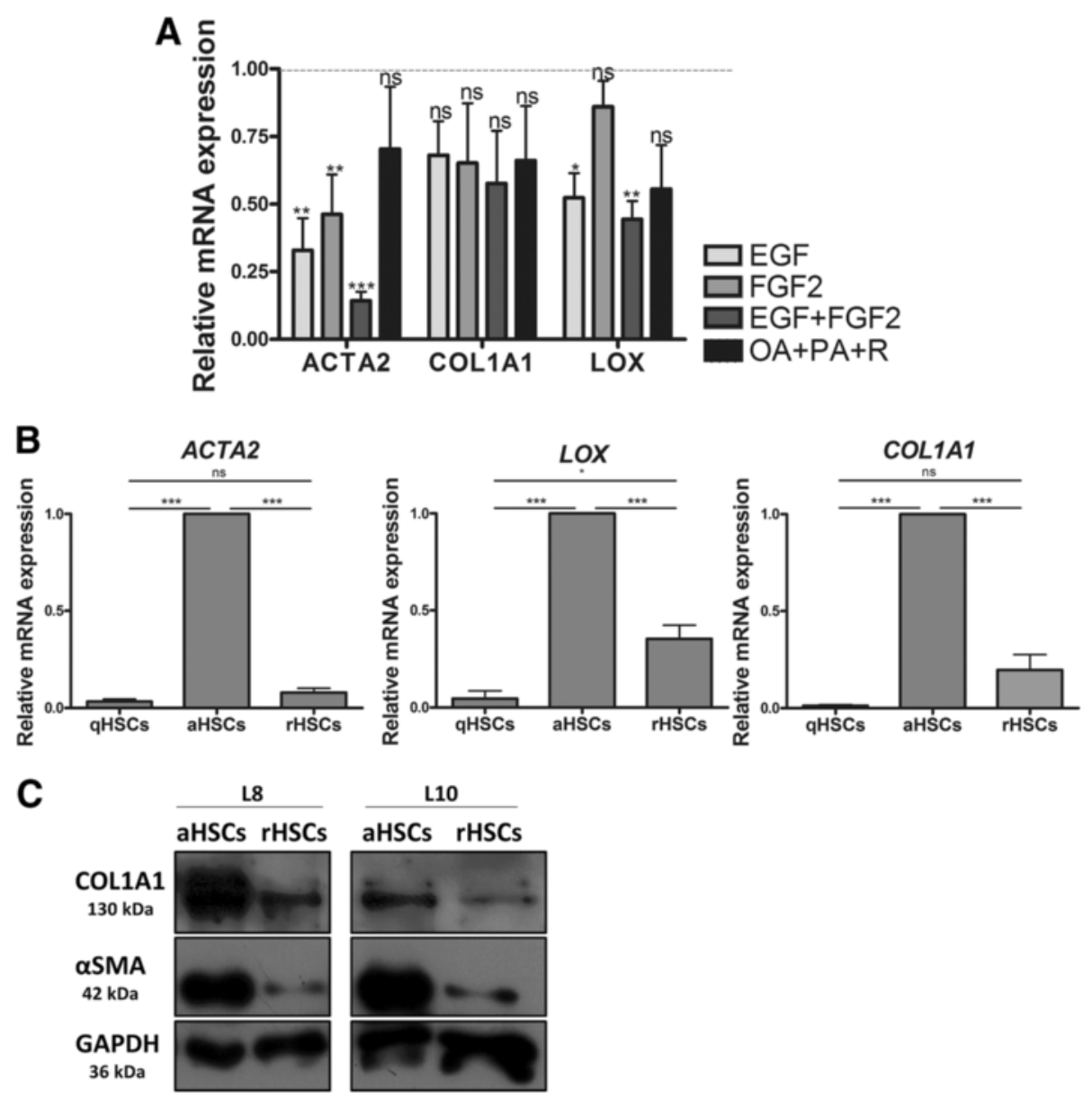

Fig. 2 EGF, FGF2, retinol, palmitic acid, and oleic acid act synergistically to negatively regulate the expression of ACTA2, COLIA1, and LOX in human primary HSCs. a Human aHSCs were exposed to recombinant human EGF (20 ng/mL), FGF2 (10 ng/mL), a combination of both, or a

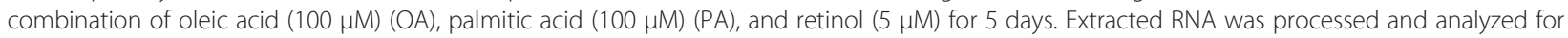
ACTA2, COL1A1, and LOX expression by RTq-PCR. Results are presented as relative fold change to untreated control cells (dotted line). b mRNA expression levels of ACTA2, COLIA1, and LOX in non-cultured quiescent (q), culture activated (a), and reverted (r) HSCs incubated for 5 days with RM (20 ng/mL EGF, $10 \mathrm{ng} / \mathrm{mL}$ FGF2, $100 \mu \mathrm{M}$ OA, $100 \mu \mathrm{M}$ PA, $5 \mu \mathrm{M}$ R) from three different (corresponding) donors. The expression levels are presented as relative fold change to aHSCs. The results presented are from three to five different donors. In the graphs, the results are displayed as means \pm SEM. ns not significant, $p \geq 0.05,{ }^{*} p<0.05,{ }^{* *} p<0.01,{ }^{* * *} p<0.001$. c a-SMA, COL1A1, and GAPDH protein levels in aHSCs and rHSCs from corresponding donors 


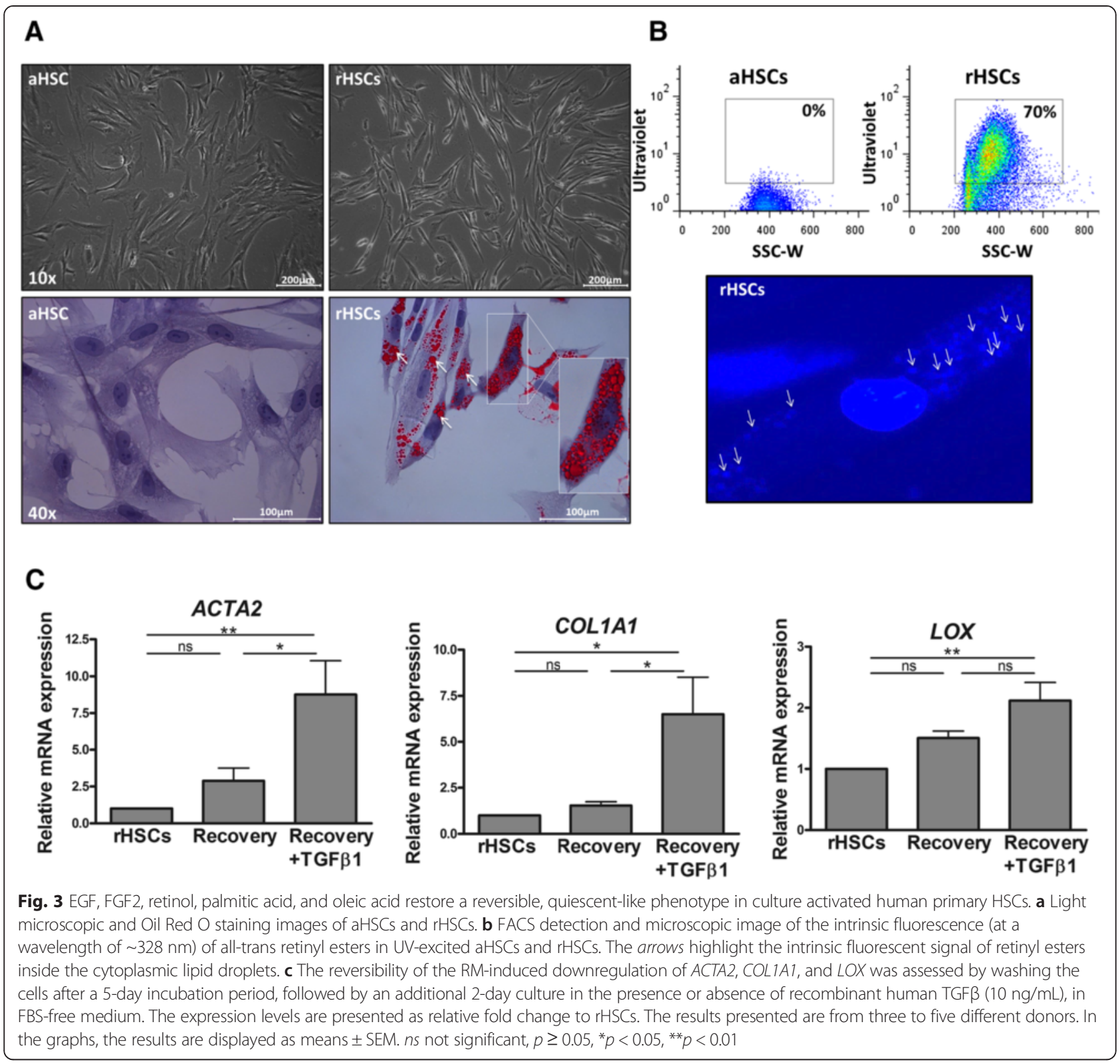

them remain $\mathrm{UV}^{-}(\sim 30 \%)$ (Fig 3b). To assess whether this difference in ability to store vitamin A is linked to a difference in activation status, FACS-sorted $\mathrm{UV}^{+}$and $\mathrm{UV}^{-}$ rHSCs were analyzed for the expression of activation markers but no difference was observed (Additional file 1: Figure S3). Interestingly, the ability of RM to revert the activated phenotype is similarly shown in a HSC-derived myofibroblast population isolated from a cirrhotic patient (Additional file 1: Figure S4). To investigate whether this induced quiescent-like state is reversible, we allowed the cells to recover for 2 days in the absence or presence of transforming growth factor beta (TGF $\beta$ ). In the absence of TGF $\beta$, the cells maintain low expression levels of profibrogenic genes (Fig. 3c). However, in the presence of
TGF $\beta$, the cells again upregulate the expression of ACTA2, COL1A1, and LOX (Fig. 3c). A viability assay was performed to rule out that the observed effects are related to cytotoxic effects of RM (Additional file 1: Figure S5A). Moreover, a 3-day recovery period with $10 \%$ fetal bovine serum (FBS) supplemented culture medium also significantly increases the expression of ACTA2 and LOX in rHSCs (Additional file 1: Figure S5B).

To evaluate whether RM can, in addition to revert, also actively prevent the culture-induced activation of HSCs, freshly isolated mouse qHSCs were seeded in normal control conditions or RM optimized for mouse HSC cultures (mRM; see "Methods") until day 6. We find that the cells grown in mRM maintain low 
expression levels of Acta2, Col1a1, and Lox, similar to uncultured D0 mouse qHSCs and have an intermediary phenotype with large intra-cytoplasmic lipid droplets (Additional file 1: Figure S6).

\section{In vitro reverted human HSCs have a reduced proliferation and matrix metalloproteinase activity}

It is known that aHSCs functionally differ from qHSCs by their increased proliferation, unbalanced ECM homeostasis, and higher migratory potential [4]. Therefore, we further investigated whether human in vitro rHSCs functionally differ from their activated counterparts by comparing their proliferation (EdU incorporation), ECM degradation (in situ zymography), and PDGF-BB-induced migration (transwell assay) (Fig. 4). We show that RM significantly reduces the proliferation (Fig. 4a-c) and matrix metalloproteinase (MMP) activity of human aHSCs (Fig. 4d, e). Furthermore, while PDGF-BB and TGF $\beta$ increase proliferation and protease activity in aHSCs, respectively, the stimulatory effect of both growth factors is totally abrogated in rHSCs (Fig. 4a-e). Although we note a clear difference in basal, non-stimulated migration between aHSCs and rHSCs (Fig. 4f), the differences are not significant and presumably linked to the differences in proliferation. Moreover, PDGF-BB-induced migration was comparable in both groups (Fig. 4f).

\section{The overall gene expression profile of in vitro rHSCs is distinct from that of aHSCs and qHSCs}

To gain more insight into the gene expression changes elicited by this transient reversion into a quiescent-like state, the global gene expression profile of rHSCs was assessed and compared to that of freshly isolated, noncultured qHSCs and culture aHSCs using the human genome U219 arrays. We find that the global gene expression profile of rHSCs resembles more closely that of aHSCs than qHSCs (Fig. 5a), with 2277 (rHSC vs aHSC) against 9122 (rHSC vs qHSC) genes expressed at significantly different levels (Student $t$ test, $p \leq 0.05$ ) (Fig. 5b). We find that many of the top upregulated genes in rHSCs compared to aHSCs are inflammationrelated, i.e., $I L-8, I L-33, I L-1 \beta, C X C L 1, C X C L 2$, and CXCL6, and over $60 \%$ of the $\geq 2$-fold deregulated genes in rHSCs are downregulated and include genes such as TNNT2, SULT1E1, and ACTG2 (Fig. 5c, d). We were further interested in identifying potential molecular processes governing this phenotypic reversion of the activated state. We find that although a limited number of genes $(n=375)$ is at least twofold differentially regulated upon RM exposure (Fig. 6a), around $75 \%(n=279)$ of these genes are also deregulated upon activation (Fig. 6a, Additional file 2: Table S1) and 212 genes have an expression profile that negatively correlates with changes observed during HSC activation (Fig. 6b, c and Fig. 6e, f). Over $50 \%$ of those genes are upregulated during activation and again downregulated in rHSCs (Fig. 6b, c). This set includes many well-known pro-fibrogenic genes, i.e., COL5A1, ADAM12, LOXL1, ACTG2, NOTCH3, and $C R Y A B$, and associates with Gene Ontology (GO) terms such as "Extracellular matrix organization," "Actin binding," and "Muscle contraction" (Fig. 6d). Interestingly, this set of genes is enriched for different KEGG pathways of major importance in aHSCs, including "Vascular smooth muscle contraction" ( $p$ value $8.28 \times 10^{-8}$ ), "Focal adhesion" $\left(6.48 \times 10^{-5}\right)$, "Regulation of actin cytoskeleton" $\left(9.47 \times 10^{-5}\right)$, and "ECM receptor interaction" $\left(2.13 \times 10^{-3}\right)$ (Fig. 6d). Another $23 \%$ of the genes show the opposite negative correlation, i.e., downregulated during activation and upregulated in rHSCs (Fig. 6e, f). This set is associated with more general GO terms and enriches for genes pertaining to "Retinol metabolism" (Fig. 6g).

\section{In vitro reverted human HSCs upregulate the expression} of in vivo inactivated-HSC-specific markers

To determine whether human HSCs reverted to quiescence-like by RM share characteristics with in vivo inactivated HSCs (iaHSCs) found in the mouse liver after recovery from experimentally induced fibrosis $[13,14]$, we measured the expression of a panel of genes $(n=10)$ previously identified as upregulated or specific for in vivo iaHSCs compared to aHSCs and qHSCs [13, 15], in aHSCs and $r$ HSCs from three HSC donors. Although there was inter-donor variation, we find all 10 genes to be consistently upregulated in rHSCs (Table 2).

\section{Discussion}

A strong body of evidence suggests that upon removal of the etiological agent, liver fibrosis can spontaneously revert in patients with secondary biliary fibrosis [20], hepatitis B [21] and C [22], non-alcoholic steatohepatitis [23], and autoimmune hepatitis [24]. Recent studies in rodent models of liver fibrosis have convincingly shown that fibrosis regression is in part driven by the reversion of aHSCs to quiescence-like HSCs [13, 14]. While this ability to revert was shown both in vitro [11, 12] and in vivo $[13,14]$ for rodent HSCs, it remains unknown whether the activated phenotype of human HSCs can be reverted as well. Moreover, the molecular mechanisms underlying the intermediate phenotype of reverted HSCs remain largely unknown. Therefore, our study aimed at identifying conditions that can revert the activated status of human HSCs and to map the molecular events associated with this phenotype reversion.

Our experiments reveal a role for EGF and FGF2 in negatively regulating the activated phenotype of human HSCs. The identification of EGF as a negative regulator of HSC activation is counterintuitive. Indeed, EGF is 


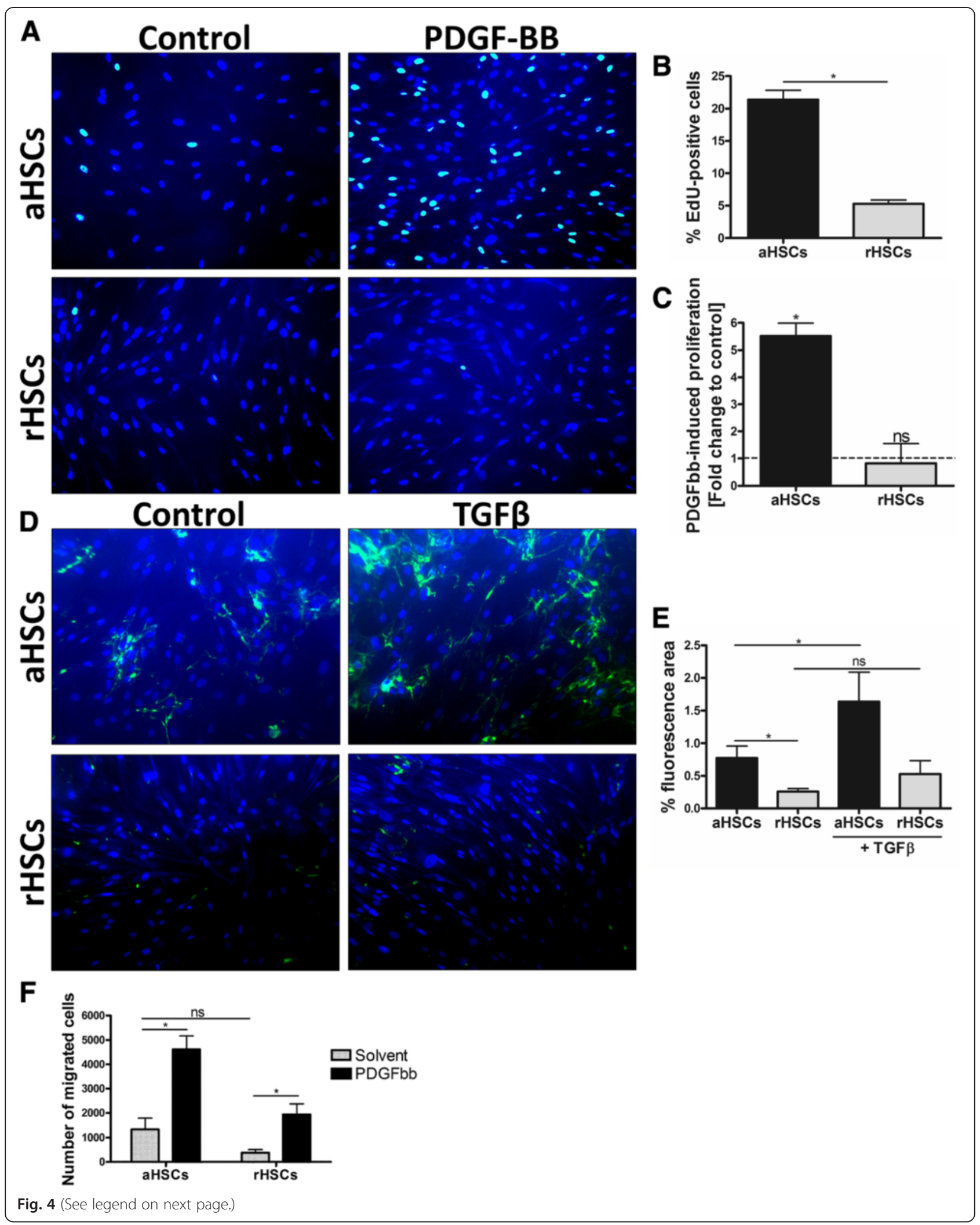


(See figure on previous page.)

Fig. 4 Functional comparison of human aHSCs and rHSCs. a EdU staining images showing the influence of RM on basal and PDGF-BB-induced HSC proliferation. $\mathbf{b}$ The percentage proliferative cells, calculated as the ratio of EdU-positive nuclei over the total (DAPI) nuclei. $\mathbf{c}$ The PDGF-BB (20 ng/mL) induced fold change in proliferation for aHSCs and rHSCs, relative to their basal condition. $\mathbf{d}$ Fluorescent images depicting the basal and TGF $\beta$-induced $(10 \mathrm{ng} / \mathrm{mL}$ ) proteolytic digestion (green) of the fluorescein-labeled gelatin substrate by aHSCs and rHSCs. e The quantified basal and TGF $\beta$-induced matrix metalloproteinase activity of aHSCs and rHSCs presented as the percentage green stained area over the total image area. f The PDGF-BBinducible migration of aHSCs and rHSCs was assessed in a transwell migration assay. The aHSCs and rHSCs were seeded in collagen-coated Boyden chambers and stimulated with PDGF-BB $(20 \mathrm{ng} / \mathrm{mL})$ or with its solvent as a control, in the lower compartment. Results are presented as the number of migrated cells in each condition. The presented results are from three different donors. ns not significant, $p \geq 0.05,{ }^{*} p<0.05$

overexpressed in the fibrotic liver [25], plays a central role during liver regeneration [26], and is member of a 186-gene signature predictive of progressive cirrhosis, hepatocellular carcinoma, and death in patients with cirrhosis [27]. Moreover, the pharmacological inhibition of the EGF receptor was shown to attenuate liver fibrosis [28]. However, to our knowledge, the present study is the first one to address the direct effect of EGF stimulation on the expression of activation markers in human primary HSCs. We find that FGF2, another growth factor known to be overexpressed in the fibrotic liver [29], synergized the negative regulation of ACTA2 and LOX expression by EGF. Although this synergistic effect was not very pronounced, it was consistent in HSCs isolated from patients with different backgrounds (i.e., age and gender). A synergy between EGF and FGF2 in the negative regulation of human HSC activation has not been shown yet; however, a previous study already showed that EGF and FGF2 can act synergistically to increase the proliferation of neural precursor cells [30].
A

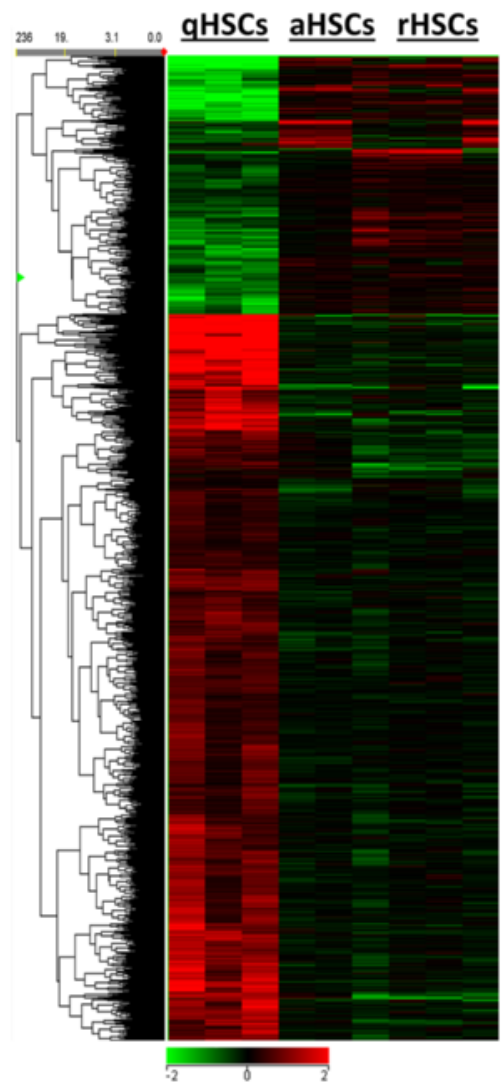

B

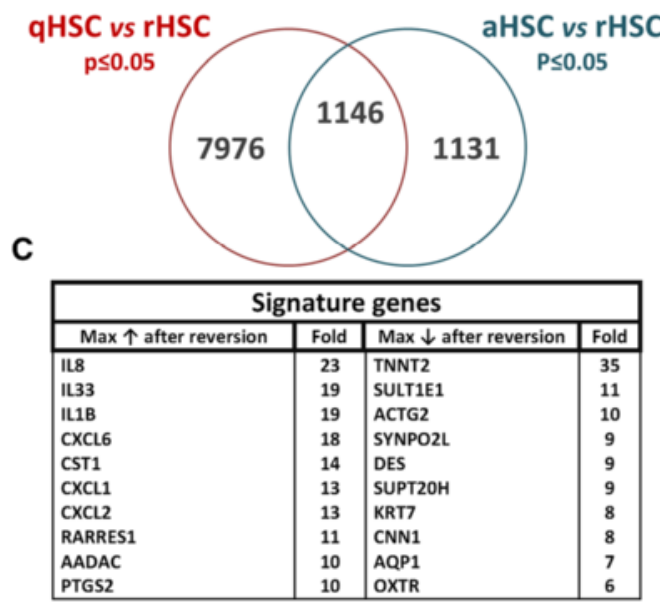

D

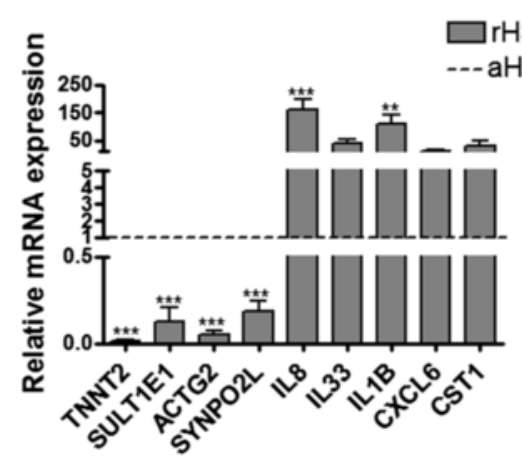

Fig. 5 Gene expression changes elicited during the in vitro reversion of human HSC activation. a Heatmap representing the hierarchical clustering of genes significantly differentially regulated between aHSCs, qHSCs, and rHSCs. $\mathbf{b}$ Venn diagram showing the number of genes significantly differentially regulated between qHSCs and rHSCS or aHSCs and rHSCs. The intersection of the Venn diagram shows the number of overlapping genes significantly differentially regulated in both comparisons. $\mathbf{c}$ List of fold changes (relative to aHSCs) for the top 10 genes upregulated and downregulated in rHSCS (microarray data). $\mathbf{d}$ Confirmation of the expression levels of selected top deregulated genes by RTq-PCR. The presented results are from three different donors. ${ }^{* *} p<0.01,{ }^{* * *} p<0.001$ 

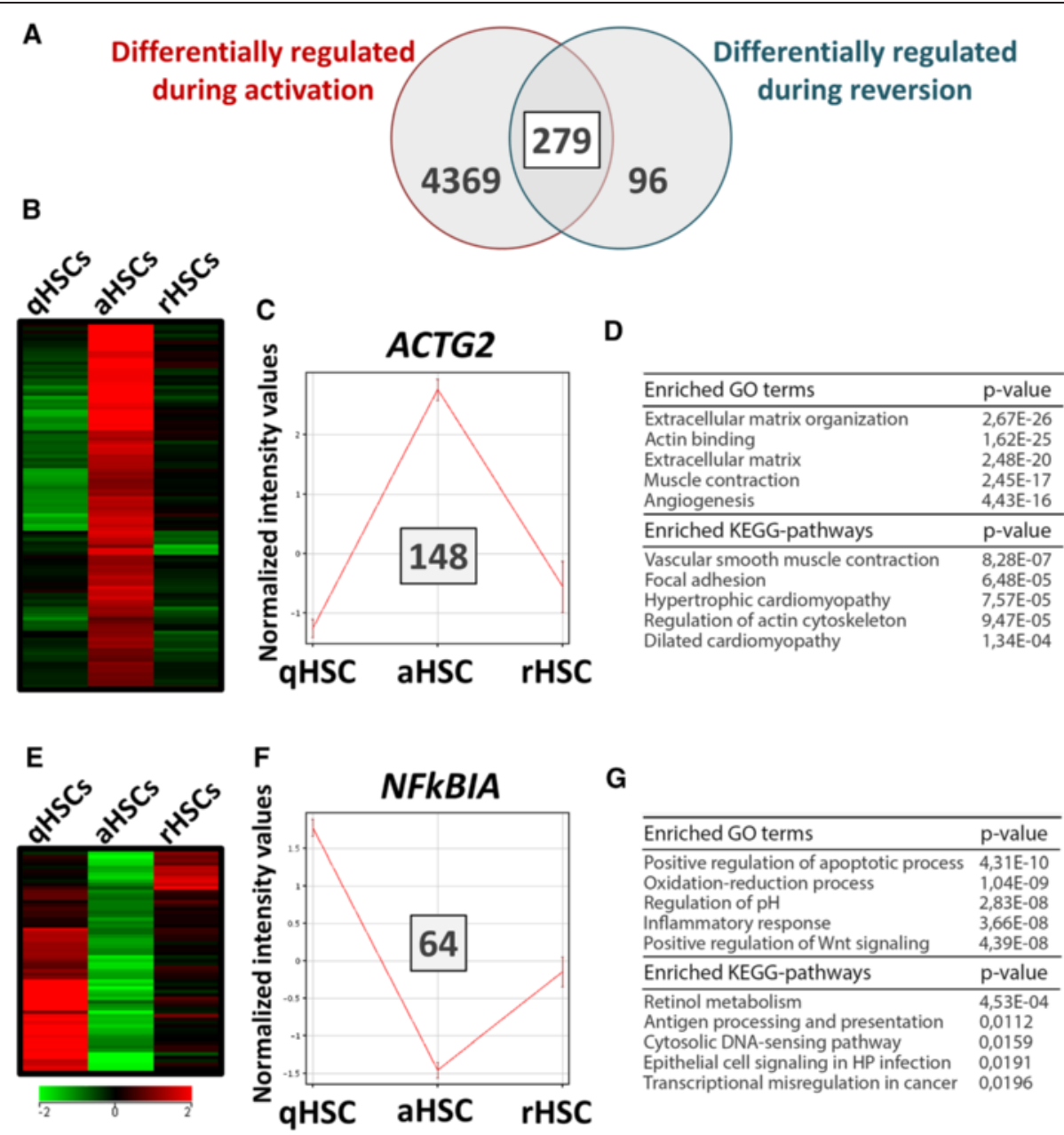

G

\begin{tabular}{ll}
\hline Enriched GO terms & $\mathrm{p}$-value \\
\hline Positive regulation of apoptotic process & $4,31 \mathrm{E}-10$ \\
Oxidation-reduction process & $1,04 \mathrm{E}-09$ \\
Regulation of pH & $2,83 \mathrm{E}-08$ \\
Inflammatory response & $3,66 \mathrm{E}-08$ \\
Positive regulation of Wnt signaling & $4,39 \mathrm{E}-08$ \\
\hline Enriched KEGG-pathways & $\mathrm{p}$-value \\
\hline Retinol metabolism & $4,53 \mathrm{E}-04$ \\
Antigen processing and presentation & 0,0112 \\
Cytosolic DNA-sensing pathway & 0,0159 \\
Epithelial cell signaling in HP infection & 0,0191 \\
Transcriptional misregulation in cancer & 0,0196 \\
&
\end{tabular}

Fig. 6 Gene expression changes during in vitro reversion of human primary HSCs inversely correlate with changes observed during in vitro HSC activation. a Venn diagram showing the number of genes differentially regulated during HSC activation ([qHSCs vs aHSCs], $p \leq 0.05$ and fold change 22) and the number of genes differentially regulated during in vitro reversion to quiescence-like ([aHSCs vs rHSCs], $p \leq 0.05$ and fold change $\geq 2$ ). The intersection of the Venn diagram shows the number of overlapping genes between both comparisons. $\mathbf{b}$ Expression heatmap for the genes that are significantly upregulated during HSC activation and significantly downregulated during reversion to quiescence-like. c Profile plot showing the expression profile of ACTG2 expression in qHSCs, aHSCs, and rHSCs as illustration for the 148 genes showing a similar expression profile. $\mathbf{d}$ The top five Gene Ontology (GO) terms and KEGG pathways associated with the set of genes represented in (c). e Expression heatmap for the genes that are significantly downregulated during HSC activation and significantly upregulated during reversion to quiescent-like cells. $\mathbf{f}$ Profile plot showing the expression profile of NFKBIA expression in qHSCs, aHSCs, and rHSCs as illustration for the 64 genes showing a similar expression profile. $\mathbf{g}$ The top five $\mathrm{GO}$ terms and KEGG pathways associated with the set of genes represented in (f)

Moreover, this same study showed that FGF2 can induce EGF responsiveness, providing a possible explanation for our observation. We also find that a mix of palmitic acid and retinol induces the formation of retinyl estercontaining intra-cytoplasmic lipid droplets in human HSCs. This is in concordance with previous work by Lee et al. showing that palmitate induces the upregulation of ADRP, a lipid droplet associated protein, and that this effect was potentiated by retinol in human HSCs [31]. We find that adding oleic acid further increased the number of lipid droplets and lipid droplet-positive cells induced by retinol and palmitic acid (data not shown). Interestingly, although the combination of oleic acid, palmitic acid, and retinol only has a subtle effect on the expression of activation markers, it strongly potentiates the effect of EGF and FGF2 on the reduced expression of ACTA2, COL1A1, and LOX. Although not all cells incorporated lipid droplets and around $30 \%$ of the cells remained $\mathrm{UV}^{-}$, no difference was found in the overall expression levels of the different activation markers between $\mathrm{UV}^{+}$and $\mathrm{UV}^{-}$rHSCs. This indicates that HSCs are heterogeneous in their capacity for retinoid and lipid storage, corroborating previous studies by D'Ambrosio et al. [19]. Furthermore, it suggests that the potentiated effect observed for RM is not dependent on the presence of retinyl ester-containing lipid droplets. 
Table 2 Overview of the fold difference in mRNA expression levels of in vivo iaHSC signature genes in rHSCs and aHSCs

\begin{tabular}{lllll}
\hline & & \multicolumn{3}{l}{ Fold change rHSC vs aHSC } \\
\cline { 3 - 5 } Gene & Accession & Donor L4 & Donor L8 & Donor L12 \\
\hline CXCL1 & NM_001511 & 52 & 30.7 & 26 \\
CXCL2 & NM_002089 & 39.1 & 7.3 & 23.6 \\
CXCL10 & NM_001565 & 3.7 & 32.7 & nd \\
CTSS (tv2) & NM_001199739 & 27.6 & 20.5 & 10.1 \\
LY86 & NM_004271 & 1.8 & 26.4 & 6.2 \\
CAPN6 & NM_014289 & 2.6 & 5.2 & 14.1 \\
RND1 & NM_014470 & 1.9 & 11.7 & 6.6 \\
KRT20 & NM_019010 & 6 & nd & 3.9 \\
CX3CR1 (tv1) & NM_001171174 & 3.2 & nd & 5.4 \\
GPC3 (tv4) & NM_001164619 & 1.5 & 1.8 & 1.2
\end{tabular}

tv transcript variant, nd not detected

Although our results indicate the ability of RM to revert the activated phenotype of HSCs isolated from a cirrhotic patient, the therapeutic relevance of this finding remains to be demonstrated. Indeed, the experimental use of in vivo activated human HSCs necessitates their culture expansion, during which the cells are exposed to the same artificial environment as the in vitro activated HSCs used in the present study. Although not shown for human HSCs yet, we recently demonstrated that the gene expression profile of mouse HSCs is significantly altered as soon as $10 \mathrm{~h}$ after in vitro culture $[32,33]$. Therefore, we believe that even a relatively short-term culture expansion of in vivo aHSCs will result in a deregulated gene expression profile that is not necessarily representative of the in vivo situation anymore. In addition to a reversion of the activated phenotype, we show that RM can prevent the culture-induced activation of qHSCs. Because human qHSC isolations are characterized by modest yields and freshly isolated cells are culture expanded during which they quickly acquire an activated phenotype, these experiments were performed on freshly isolated mouse qHSCs.

Microarray analysis of non-cultured human qHSCs and in vitro aHSCs and rHSCs reveals that the global gene expression profile of rHSCs was still very similar to that of aHSCs, indicating that this reversal in phenotype is not substantiated by a thorough reprogramming at the transcriptional level. Indeed, our study found only 375 genes to be differentially regulated between rHSCs and aHSCs. Although this corresponds to only $1.8 \%$ of all the genes represented on the HGU219 array, $75 \%$ of these genes are also differentially regulated during activation and over $56 \%$ show an expression profile that negatively correlates during activation and reversion. The major part of those genes is upregulated and again downregulated during activation and reversion, respectively, and is enriched for genes that are involved in pathways that are known to play major roles in aHSCs such as "Vascular smooth muscle contraction" and "Focal adhesion". Therefore, changes in expression of these genes probably underpin the observed shift in HSC phenotype. Interestingly, serum response factor (SRF) and specificity protein 1 (SP1) were predicted to be the transcription factors with the highest importance and occurrence score, respectively, for the binding of common regulatory elements for this set of genes (http://dire.dcode.org/). SRF is mainly involved in cell differentiation by regulating genes that control cell growth, cytoskeletal organization, contractility, and motility [34]. In rodent HSCs, Srf expression was shown to be inducible by TGF $\beta$ and small interfering RNA (siRNA)-mediated knockdown of Srf resulted in the downregulation of Acta2 expression [35]. SP1, a member of the Krüppel-like family that was shown to have an increased DNA binding activity in aHSCs, co-operates with Krüppel-like factor 6 (KLF6) to induce the expression of TGF $\beta$ and urokinase plasminogen activator $(u P A)$, which promotes the activation of latent TGF $\beta$ [36, 37]. Other pro-fibrogenic genes regulated by SP1 include Col1a2 and Timp1 [38, 39]. In rodent models of kidney, lung, and liver fibrosis, SP1 targeting was shown to be a powerful anti-fibrotic strategy [40]. This anti-fibrotic effect is presumably due to the abrogation of SP1 activity in myofibroblasts, as it was shown that SP1 targeting inhibits the expression of profibrogenic genes as well as proliferation in rat aHSCs [41]. Future research will show whether targeting of both these transcription factors in fibrotic patients might represent a therapeutic strategy to revert the activated phenotype of human HSCs.

In inverse analogy to the differentiation of adipocytes from pre-adipocytes, anti-adipogenic regulation has been shown to underlie HSC activation [42]. Because both oleic acid and palmitic acid are reported to stimulate adipocytic differentiation [43], we speculate that they potentiate the negative effect of EGF and FGF2 on the expression of activation markers by enforcing the transdifferentiation of human aHSCs towards the more adipogenic qHSC phenotype. The loss of PPAR $\gamma$ activity, the master regulator of adipogenesis, has been found to play an important role during HSC activation [44-46], and overexpression in activated HSCs has been shown to induce a phenotypic switch to a more quiescent-like phenotype [11]. Surprisingly, the adipogenic phenotype of rHSCs was not supported by an increased expression of PPARY and its downstream effector genes. Recent studies show that PPARY induction is a multistep process, necessitating both epigenetic reconfiguration [47, 48] and chromatin remodeling [49]. Furthermore, transcriptional activities of PPAR $\gamma$ have been shown to require chromatin presetting [50], and in turn, PPAR $\gamma$ can lead to post-translational histone 
modifications (for review, see [51]) to further drive and maintain the adipogenic transcription program. Seemingly, the adipogenic cue provided by RM or the treatment duration is an insufficient trigger for this multistep process to take place in activated primary human HSCs. Nevertheless, we find that RM induces the expression of ADRP (Additional file 1: Figure S7), a protein known to be expressed early during adipogenesis and previously shown to be sufficient to stimulate lipid droplet formation and accumulation independently of PPARY or other lipogenic genes [52]. Moreover, work by Lee et al. previously demonstrated a role for palmitic acid and retinol in upregulating the expression of ADRP [53] in LX-2 cells, an immortalized human HSC line. The authors report that the treatment correlated with reduced ACTA 2 and COL1A1 expression and ADRP knockdown abrogated the treatment's effect [53]. In contrast, our experiments do not reveal a direct role for ADRP in the negative regulation of activation genes. Nevertheless, increased $A D R P$ expression provides a possible explanation for the potentiating effect of the dietary supplements on the downregulation of ACTA2, COL1A1, and $L O X$ by EGF and FGF2.

At the functional level, we show the RM's potency to not only reduce basal proliferation and MMP activity of the cells, but also to actively counteract the increased functionality of HSCs by well-known pro-fibrogenic factors. The mechanism underlying these effects however remains unknown and needs further attention.

Recently, Kisseleva et al. for the first time described the unique phenotypic characteristics of murine in vivo iaHSCs and identified a set of genes highly expressed in iaHSCs compared to qHSCs and/or aHSCs [13, 15]. Interestingly, we find that these genes were consistently upregulated in rHSCs, derived from different donors. Although the relevance of this observation remains to be demonstrated, their increased expression in in vitro reverted human HSCs suggests also a potential role for these genes in promoting a quiescent-like phenotype in human HSCs.

\section{Conclusions}

In conclusion, this study for the first time provides evidence that human HSCs can be reverted to a quiescentlike state and provides a first phenotypic and genomic characterization of in vitro reverted human HSCs.

\section{Methods}

\section{Patient samples}

The protocol and experiments were approved by the ethical committees of St-Luc Hospital and the Faculty of Medicine of Université Catholique de Louvain. An agreement from the Belgian Ministry of Health was obtained for the Hepatocytes and Hepatic Stem Cells Bank. A written and signed informed consent has been obtained for each human liver used in the current study. Five livers, for which the clinical characteristics are summarized in Table 1, were used in the current study. Additionally, commercially available primary human liver myofibroblasts derived essentially from hepatic stellate cells and portal fibroblasts (Tebu-Bio NV, Antwerp, Belgium) was used.

\section{Isolation and in vitro reversion of human activated HSCs}

Human aHSCs were obtained by plating the qHSCenriched population obtained after Nycodenz (Myegaard, Oslo, Norway) gradient centrifugation of the nonparenchymal cell fraction [54]. Homogeneous populations of aHSCs were obtained after three passages in Dulbecco's modified Eagle's medium (DMEM) supplemented with $10 \% \mathrm{FBS}$ at $37{ }^{\circ} \mathrm{C}$ in a humidified atmosphere with $5 \%$ $\mathrm{CO}_{2}$ and subsequently cultured in $1 \%$ FBS supplemented medium. Prior to each passage, cells were washed with phosphate buffered saline (PBS) and lifted using $0.05 \%$ trypsin (Lonza). For reversion of the activated phenotype, human aHSCs were seeded at a density of 10,000 cells/ $\mathrm{cm}^{2}$ (unless stated differently), and $24 \mathrm{~h}$ later, the cells were washed and incubated with DMEM supplemented with $1 \%$ FBS, $20 \mathrm{ng} / \mathrm{mL}$ epidermal growth factor (EGF) (Peprotech, London, UK), $10 \mathrm{ng} / \mathrm{mL}$ fibroblast growth factor 2 (FGF2) (Peprotech), $100 \mu \mathrm{M}$ oleic acid (Sigma), $100 \mu \mathrm{M}$ palmitic acid (Sigma), and $5 \mu \mathrm{M}$ retinol (Sigma). The medium was refreshed every 2 days for 5 days, and the cells were harvested for further analysis on day 6 .

\section{Isolation of non-plated human quiescent HSCs}

The human liver parenchymal and non-parenchymal cell fractions were separated from each other by sequential perfusion of liver pieces with pre-warmed EGTAcontaining EBSS medium (Lonza, Verviers, Belgium) and a digestion enzyme solution (EBSS supplemented with $0.9 \mathrm{mg} / \mathrm{mL}$ collagenase $\mathrm{P}$ and $0.03 \mathrm{mg} / \mathrm{mL}$ soybean trypsin inhibitor (Roche)) for 9 to $12 \mathrm{~min}$. Collagenase digestion was stopped with ice-cold M199 wash medium (Lonza) containing $0.03 \mathrm{mg} / \mathrm{mL}$ of soybean trypsin inhibitor and $100 \mathrm{~mL} / \mathrm{L}$ of human plasma [55]. After filtration, the non-parenchymal cells were separated from the parenchymal cells by subsequent low-speed centrifugation steps $(50 g)$ and submitted to an additional centrifugation step $(640 g)$. Non-parenchymal cell pellets were then resuspended and cryopreserved in DMEM (Lonza) supplemented with 20 \% FBS (Biochrom GmbH, Berlin, Germany) and $5 \%$ dimethyl sulfoxide (DMSO) (Sigma, St. Louis, MO, USA). The isolation of highpurity human qHSCs was performed as described previously [56]. In brief, dissociated and washed single nonparenchymal cells were suspended in a $5 \%$ FBS, $2 \mathrm{mM}$ 
EDTA (Sigma) buffer and incubated for $30 \mathrm{~min}$ at $4{ }^{\circ} \mathrm{C}$ with anti-CD32 (Abcam, Cambridge, UK) and anti-CD45 (BD Biosciences, San Jose, CA, USA). 7-Aminoactinomycin (7-AAD) (eBioscience, San Diego, CA, USA) was used for the exclusion of non-viable cells. Pure populations of qHSCs were sorted as $\mathrm{CD} 32^{-} \mathrm{CD} 45^{-} \mathrm{UV}^{+}$cells, using FACSAria $^{\text {Tx }}$ (BD Biosciences). RNA from freshly isolated qHSCs was obtained using RNeasy Micro Kit (Qiagen). RNA samples were amplified using the Ovation Pico WTA system V2 (NuGEN).

Mouse HSCs were isolated from male BalbC mice (age 20-25 weeks) (Charles River Laboratories, Larbresle, France) and cultured in DMEM with $10 \%$ FBS, as described previously [57]. For treatment experiments with mouse HSC reverting medium (mRM) (DMEM supplemented with $10 \% \mathrm{FBS}, 40 \mathrm{ng} / \mathrm{mL}$ EGF, $20 \mathrm{ng} / \mathrm{mL}$ FGF2, $100 \mu \mathrm{M}$ oleic acid, $100 \mu \mathrm{M}$ palmitic acid, and $5 \mu \mathrm{M}$ retinol), freshly isolated mouse qHSCs were seeded at a density of 7.500 cells $/ \mathrm{cm}^{2}$. The inhibition of activation was assessed by relative comparison to control cells cultured in DMEM supplemented with $10 \%$ FBS for the same period of time. All procedures on animals were carried out in accordance with the University's guidelines for the care and use of laboratory animals in research. The performed experiments were approved by the ethical committee of the Vrije Universiteit Brussel in project 12-212-1.

\section{Gene expression profiling and analysis}

Total RNA originating from cultured (activated and reverted HSCs; $n=3$ from corresponding donors) and uncultured (FACS-sorted quiescent HSCs; $n=3$ from two corresponding donors) primary human HSCs was isolated by using RNeasy Micro Kit (Qiagen GmbH, Hilden, Germany) following the manufacturer's recommendations. Total RNA concentration and quality control was assessed using RNA 6000 Pico Kit (Agilent, Santa Clara, CA, USA). RNA samples were amplified by using Ovation PicoSL System V2 (NuGene Technologies, CA, USA) and ENCORe Biotin module (NuGene Technologies). Amplified and purified RNA samples were labeled and hybridized to the Affymetrix HG-U219 GeneChip (Affymetrix, Santa Clara, California, USA). Data normalization and analysis was performed using GeneSpring GX12 (Agilent, Santa Clara, CA, USA) as described previously [32]. Briefly, Affymetrix gene expression data were normalized using the robust multi-array algorithm [58]. For the detection of differentially expressed genes, a $p$ value cutoff of 0.05 was used in combination with a fold change cutoff of 2.0. Functional analysis of gene expression data (gene ontology (GO) and Kyoto Encyclopedia of Genes and Genomes (KEGG) pathways) was conducted using an open access, high-level cross-platform microarray dataset analysis tool (InCroMAP) (http://www.ra.cs.uni-tuebingen.de/software/
InCroMAP/). Raw data are made publically available on the NCBI Gene Expression Omnibus database, with accession number GSE68001.

\section{Immunocytochemistry}

Nycodenz-isolated HSCs cultured for 1 day or for three passages were washed with PBS and fixed for $10 \mathrm{~min}$ with $4 \%$ buffered formaldehyde (Merck, Darmstadt, Germany). Following permeabilization with $0.1 \%$ Triton X-100 (in PBS containing $1 \%$ bovine serum albumin), cells were incubated overnight with anti- $\alpha$ SMA (1/1000) (Sigma). Primary antibody binding was visualized using an Alexa488-labeled secondary antibody (1/200) (Invitrogen, Eugene, OR, USA). Images were taken with an AxioCam MRc5 digital camera (Carl Zeiss). NCAM1, nestin, and desmin stainings were performed as described previously [59]. Briefly, the cells were fixed, endogenous peroxidase was eliminated, and cells were permeabilized using PBS containing $1 \%$ Triton X-100 (Sigma). Thereafter, the cells were blocked by 1 -h incubation in PBS containing $1 \%$ bovine serum albumin (Sigma), incubated with primary anti-NCAM1 (1/100) (Abcam), anti-Nestin (1/1000) (Abcam), or anti-Desmin (1/50) (Abcam) for $1 \mathrm{~h}$, washed and incubated with secondary antibody (Envision, Dako) for $30 \mathrm{~min}$. Detection was performed after 5 min incubation with liquid DAB and substrate chromogen (Dako). Counterstaining was performed using Mayer's hematoxylin for $10 \mathrm{~min}$. Preparations were then mounted for microscopic analysis (DMIL, Leica, Belgium).

\section{Lipid staining}

The cells were washed with PBS and fixed in $10 \%$ formalin solution. The cells were then washed with deionized water, incubated with isopropanol for $5 \mathrm{~min}$, and stained with diluted (3/2 in water) and filtered Oil Red O $(0.3 \%(w / v)$ in $99 \%$ isopropanol) (Sigma) for $20 \mathrm{~min}$. The red-stained lipid droplets were visualized with light microscopy (Carl Zeiss).

\section{Western blot}

Cells were washed with ice-cold PBS and scraped with ice-cold lysis buffer $(170 \mathrm{mM} \mathrm{NaCl}, 10 \mathrm{mM}$ EDTA, $50 \mathrm{mM}$ Tris pH 7.4, $50 \mathrm{mM} \mathrm{NaF}, 0.2 \mathrm{mM}$ dithiothreitol, and $0.5 \% \mathrm{NP}-40$ ) supplemented with protease (Roche Diagnostics, Mannheim, Germany) and phosphatase (Roche Diagnostics) inhibitors. Protein concentrations were determined using the BCA protein assay kit (Pierce Chemical Co, Rockford, IL, USA). Fifteen microgram of protein was separated on a $8 \%$ Tris-glycine SDSpolyacrylamide gel and electroblotted onto polyvinylidene difluoride membranes (Amersham Biosciences, Little Chalfront, UK) using a wet blotting apparatus (Mini Trans-Blot Cell, BioRad, Nazareth, Belgium). Blots 
were blocked with $5 \%$ milk powder in Tris buffered saline (TBS) with $0.2 \%$ Tween (Sigma) and subsequently incubated overnight with primary anti-PDGFR $\beta$ (diluted 1/1000 in blocking buffer) (Abcam), anti- $\alpha$ SMA (1/50) (Dako), anti-COL1A1 (1/1000) (Abcam), or antiGAPDH (diluted 1/30,000) (Abcam). The membranes were washed and incubated with a horseradish peroxidaseconjugated secondary antibody $(1 / 20,000)$ (Dako, Glostrup, Denmark) for $1 \mathrm{~h}$, and the antigen was visualized by enhanced chemiluminescence using ECL substrate (Pierce Chemical Co.).

\section{Proliferation assay}

Cell proliferation of human HSCs was assessed with the Click-iT EdU Cell Proliferation Assay Kit (Invitrogen, Eugene, OR, USA). HSCs were cultured under control conditions or in RM for 6 days. On day 6, the cells were labeled with $10 \mu \mathrm{M}$ EdU for $6 \mathrm{~h}$ and subsequently formalin fixed and mounted with Prolong Gold antifade reagent with DAPI (Invitrogen). EdU incorporation was visualized according to the manufacturer's instructions. The percentage proliferation was calculated as the ratio of EdU-positive cells to DAPI-positive cells. For the PDGF-BB stimulation experiments, the control medium and RM were supplemented with $20 \mathrm{ng} / \mathrm{mL}$ human recombinant PDGF-BB for $48 \mathrm{~h}$ prior to EdU labeling. The quantification was performed on at least 500 cells per donor and is presented as the mean percentage measured in three different donors.

\section{In situ zymography}

The matrix metalloproteinase activity of human HSCs was assessed using the highly quenched, fluoresceinlabeled pig skin gelatin ( $\mathrm{DQ}^{\mathrm{in}}$ gelatin) (Invitrogen). Upon proteolytic digestion, its green fluorescence is revealed and can be used to measure enzymatic activity. A $1 \mathrm{mg} / \mathrm{mL}$ stock solution of DQ gelatin was prepared using deionized water and stored at $4{ }^{\circ} \mathrm{C}$. Prior to cell seeding $\left(20,000\right.$ cells $\left./ \mathrm{cm}^{2}\right)$, glass coverslips (12 $\mathrm{mm}$ diameter) were coated with $50 \mu \mathrm{g}$ DQ gelatin for $1 \mathrm{~h}$. Twenty-four hours post-seeding, the cells were washed with serum-free DMEM and cultured for 6 days under control conditions or RM. On day 6, the cells were formalin fixed and mounted with Prolong Gold antifade reagent with DAPI. The percentage green stained area was calculated using ImageJ (http://imagej.nih.gov/ij/ index.html). For the TGF $\beta$ stimulation experiments, the control medium and RM were supplemented with $10 \mathrm{ng} / \mathrm{mL}$ human recombinant TGF $\beta$ for $48 \mathrm{~h}$ prior to being fixed and analyzed. The quantification was performed on 5-10 images per donor and is presented as the mean percentage measured in five different donors.

\section{Migration assay}

Both aHSCs and rHSCs were seeded in collagen-coated Boyden chambers (Millipore) $(40,000$ cells/chamber) in serum-free DMEM. After $60 \mathrm{~min}$, the chambers were transferred to wells with $20 \mathrm{ng} / \mathrm{mL}$ PDGF-BB (R\&D Systems, Minneapolis, MN, USA) or its solvent as a control. After $16 \mathrm{~h}$, non-migrated cells were cleared and migrated cells were fixed with ice-cold $100 \%$ methanol and mounted with Prolong Gold antifade reagent with DAPI. The quantification was performed by manually counting the totality of migrated cells on images covering the entire membrane and is represented as the mean of three different donors.

\section{Viability/cytotoxicity assay}

For the LIVE/DEAD viability/cytotoxicity assay (Life Technologies), aHSCs and rHSCs were incubated with PBS containing $4 \mu \mathrm{M}$ ethidium homodimer and $2 \mu \mathrm{M}$ calcein for $15 \mathrm{~min}$ at $37{ }^{\circ} \mathrm{C}$, washed thoroughly with PBS, and subsequently analyzed under a fluorescent microscope (Carl Zeiss). Images were taken with an AxioCam MRc5 digital camera (Carl Zeiss).

\section{RNA purification and RTq-PCR}

RNA was extracted and purified from cultured and uncultured cells using the ReliaPrep RNA Cell Miniprep System (Promega, Madison, WI, USA). Total RNA was converted to cDNA by reverse transcription using the Revert Aid Kit (Thermo Fisher Scientific, St. Leon-Rot, Germany). Quantitative real-time polymerase chain reaction was performed using the GoTaq qPCR Master Mix with BRYTE green (Promega). A 7500 real-time PCR system was used and data was analyzed using System SDS software v2.0.6 (Applied Biosystems). Fold change differences between samples were determined using the comparative $\mathrm{Ct}$ method $(\Delta \Delta \mathrm{Ct})$. The expression level of different target genes, relative to glyceraldehyde-3-phosphate dehydrogenase (GAPDH) and the calibrator, was given by 2- $\Delta \Delta \mathrm{Ct}$. Gene-specific primers were produced by Integrated DNA Technologies (Leuven, Belgium).

\section{Statistical analysis}

GraphPad Prism v4.0.0 (GraphPad Software, La Jolla, CA, USA) was used for statistical analysis. Data in the figures are expressed as means \pm SEM. Differences among groups were tested for statistical significance by Student $t$ test or analysis of variance (ANOVA) followed by Tukey's test, depending on the number of groups (ns = not significant, $\left.p \leq 0.05,{ }^{*} p<0.05,{ }^{* * *} p<0.01,{ }^{* * * * *} p<0.001\right)$.

\section{Additional files}

Additional file 1: Supplementary figures. (ZIP $4596 \mathrm{~kb})$ 
Additional file 2: List of overlapping genes at least 2-fold differentially expressed upon in vitro activation and reversion of human HSCs. (XLSX $27 \mathrm{~kb}$ )

\section{Abbreviations}

ECM: extracellular matrix; EGF: epidermal growth factor; FGF2: fibroblast growth factor 2; GO: Gene Ontology; HSC: hepatic stellate cell-activated (a), quiescent (q), reverted (r), inactivated (ia); KEGG: Kyoto Encyclopedia of Genes and Genomes; OA: oleic acid; PA: palmitic acid; R: retinol; RM: reverting medium; UV: ultraviolet.

\section{Competing interests}

The authors declare that they have no competing interests.

\section{Authors' contributions}

$A E, M N$, and $L A v G$ conceived, designed, and performed the experiments and analyzed the data. PSB provided technical assistance. AE and LAvG wrote the manuscript, and all authors revised it. All authors read and approved the final manuscript.

\section{Acknowledgements}

We thank Tom Schouteet, Jean-Marc Lazou (VUB), and Jonathan Evraerts (UCL) for technical assistance.

\section{Financial support}

The work was funded by a PhD grant from the Institute for the Promotion of Innovation through Science and Technology in Flanders (IWT-Vlaanderen) to Adil El Taghdouini, from a European Union FP7/Cosmetics Europe cofunded project (HeMiBio, ECGA \#266777) to L.A. van Grunsven, and from the Brussels region (ISRIB/"Brustem") to L.A. van Grunsven, M. Najimi, and E. Sokal.

\section{Author details}

'Liver Cell Biology Lab, Vrije Universiteit Brussel (VUB), Laarbeeklaan 103, 1090 Brussels, Belgium. 'Laboratory of Pediatric Hepatology and Cell Therapy, Institut de Recherche Expérimentale et Clinique (IREC), Université Catholique de Louvain, Brussels, Belgium. ${ }^{3}$ Institut d'Investigacions Biomèdiques August Pi i Sunyer (IDIBAPS), Barcelona, Spain.

\section{Received: 21 April 2015 Accepted: 15 July 2015}

\section{Published online: 06 August 2015}

\section{References}

1. Bataller R, Brenner DA. Liver fibrosis. J Clin Invest. 2005;115(2):209-18.

2. Wynn TA, Ramalingam TR. Mechanisms of fibrosis: therapeutic translation for fibrotic disease. Nat Med. 2012;18(7):1028-40.

3. Mederacke I, Hsu CC, Troeger JS, Huebener P, Mu X, Dapito DH, et al. Fate tracing reveals hepatic stellate cells as dominant contributors to liver fibrosis independent of its aetiology. Nat Commun. 2013;4:2823. doi:10.1038/ncomms3823.

4. Friedman SL. Hepatic stellate cells: protean, multifunctional, and enigmatic cells of the liver. Physiol Rev. 2008;88(1):125-72. doi:10.1152/ physrev.00013.2007.

5. Reynaert $H$, Urbain D, Geerts A. Regulation of sinusoidal perfusion in portal hypertension. Anat Rec. 2008;291(6):693-8. doi:10.1002/ar.20669.

6. Taura K, De Minicis S, Seki E, Hatano E, Iwaisako K, Osterreicher CH, et al. Hepatic stellate cells secrete angiopoietin 1 that induces angiogenesis in liver fibrosis. Gastroenterology. 2008;135(5):1729-38. http://dx.doi.org/ 10.1053/j.gastro.2008.07.065.

7. Geerts ABL, Wisse E. History, heterogeneity, developmental biology, and functions of quiescent hepatic stellate cells. Semin Liver Dis. 2001;21:311-35.

8. Ellis EL, Mann DA. Clinical evidence for the regression of liver fibrosis. J Hepatol. 2012:56(5):1171-80. http://dx.doi.org/10.1016/j.jhep.2011.09.024.

9. Iredale JPBRC, Pickering J, McCullen M, Northrop M, Pawley S, Hovell C, et al. Mechanisms of spontaneous resolution of rat liver fibrosis. Hepatic stellate cell apoptosis and reduced hepatic expression of metalloproteinase inhibitors. J Clin Invest. 1998;102:538-49.

10. Issa R, Williams E, Trim N, Kendall T, Arthur MJP, Reichen J, et al. Apoptosis of hepatic stellate cells: involvement in resolution of biliary fibrosis and regulation by soluble growth factors. Gut. 2001;48(4):548-57. doi:10.1136/ gut.48.4.548
11. Hazra S, Xiong S, Wang J, Rippe RA, Krishna V, Chatterjee K, et al. Peroxisome proliferator-activated receptor $\gamma$ induces a phenotypic switch from activated to quiescent hepatic stellate cells. J Biol Chem. 2004;279(12):11392-401. doi:10.1074/jbc.M310284200.

12. She H, Xiong S, Hazra S, Tsukamoto H. Adipogenic transcriptional regulation of hepatic stellate cells. J Biol Chem. 2005;280(6):4959-67. doi:10.1074/jbc.M410078200.

13. Kisseleva T, Cong M, Paik Y, Scholten D, Jiang C, Benner C, et al. Myofibroblasts revert to an inactive phenotype during regression of liver fibrosis. Proc Natl Acad Sci. 2012;109(24):9448-53. doi:10.1073/ pnas.1201840109.

14. Troeger JS, Mederacke I, Gwak GY, Dapito DH, Mu X, Hsu CC, et al. Deactivation of hepatic stellate cells during liver fibrosis resolution in mice. Gastroenterology. 2012;143(4):1073-83.e22. http://dx.doi.org/10.1053/ j.gastro.2012.06.036.

15. Liu XXJ, Brenner DA, Kisseleva T. Reversibility of liver fibrosis and inactivation of fibrogenic myofibroblasts. Curr Pathobiol Rep. 2013;1(3):209-14.

16. Cassiman D, Libbrecht L, Desmet V, Denef C, Roskams T. Hepatic stellate cell/ myofibroblast subpopulations in fibrotic human and rat livers. J Hepatol. 2002;36(2):200-9. http://dx.doi.org/10.1016/S0168-8278(01)00260-4.

17. Niki T, Pekny M, Hellemans K, De Bleser P, Van Den Berg K, Vaeyens F, et al. Class VI intermediate filament protein nestin is induced during activation of rat hepatic stellate cells. Hepatology. 1999;29(2):520-7. doi:10.1002/ hep.510290232.

18. Niki T, De Bleser PJ, Xu G, Van der Berg K, Wisse E, Geerts A. Comparison of glial fibrillary acidic protein and desmin staining in normal and CCl4-induced fibrotic rat livers. Hepatology. 1996;23(6):1538-45. doi:10.1002/hep.510230634.

19. D’Ambrosio DN, Walewski JL, Clugston RD, Berk PD, Rippe RA, Blaner WS Distinct populations of hepatic stellate cells in the mouse liver have different capacities for retinoid and lipid storage. PLoS One. 2011;6(9), e24993. doi:10.1371/journal.pone.0024993.

20. Hammel P, Couvelard A, OToole D, Ratouis A, Sauvanet A, Fléjou JF, et al. Regression of liver fibrosis after biliary drainage in patients with chronic pancreatitis and stenosis of the common bile duct. New Engl J Med. 2001;344(6):418-23. doi:10.1056/NEJM200102083440604.

21. Kweon Y-O, Goodman ZD, Dienstag JL, Schiff ER, Brown NA, Burkhardt E, et al. Decreasing fibrogenesis: an immunohistochemical study of paired liver biopsies following lamivudine therapy for chronic hepatitis B. J Hepatol. 2001;35(6):749-55. http://dx.doi.org/10.1016/S0168-8278(01)00218-5.

22. Arthur MJP. Reversibility of liver fibrosis and cirrhosis following treatment for hepatitis C. Gastroenterology. 2002;122(5):1525-8. http://dx.doi.org/10.1053/ gast.2002.33367.

23. Dixon JB, Bhathal PS, Hughes NR, O'Brien PE. Nonalcoholic fatty liver disease: improvement in liver histological analysis with weight loss. Hepatology. 2004;39(6):1647-54. doi:10.1002/hep.20251.

24. Abdalla A, Zalata K, Ismail A, Shiha G, Attiya M, Abo-Alyazeed A. Regression of fibrosis in paediatric autoimmune hepatitis: morphometric assessment of fibrosis versus semiquantitative methods. Fibrogenesis Tissue Repair. 2009;2(1):2.

25. Kömüves $L G$, Feren $A$, Jones $A L$, Fodor E. Expression of epidermal growth factor and its receptor in cirrhotic liver disease. J Histochem Cytochem. 2000;48(6):821-30. doi:10.1177/002215540004800610.

26. Olsen PS, Boesby S, Kirkegaard P, Therkelsen K, Almdal T, Poulsen SS, et al. Influence of epidermal growth factor on liver regeneration after partial hepatectomy in rats. Hepatology. 1988;8(5):992-6. doi:10.1002/hep.1840080503.

27. Hoshida Y, Villanueva A, Sangiovanni A, Sole M, Hur C, Andersson KL, et al. Prognostic gene expression signature for patients with hepatitis C-related early-stage cirrhosis. Gastroenterology. 2013;144(5):1024-30. http://dx.doi.org/ 10.1053/j.gastro.2013.01.021

28. Fuchs BC, Hoshida Y, Fujii T, Wei L, Yamada S, Lauwers GY, et al. Epidermal growth factor receptor inhibition attenuates liver fibrosis and development of hepatocellular carcinoma. Hepatology. 2014;59(4):1577-90. doi:10.1002/ hep.26898.

29. Rosenbaum J, Blazejewski S, Préaux A-M, Mallat A, Dhumeaux D, Mavier P. Fibroblast growth factor 2 and transforming growth factor $\beta 1$ interactions in human liver myofibroblasts. Gastroenterology. 1995;109(6):1986-96. doi:10.1016/0016-5085(95)90767-x.

30. Ciccolini F, Svendsen CN. Fibroblast growth factor 2 (FGF-2) promotes acquisition of epidermal growth factor (EGF) responsiveness in mouse striatal precursor cells: identification of neural precursors responding to both EGF and FGF-2. J Neurosci. 1998;18(19):7869-80. 
31. Lee K-W, Thiyagarajan V, Sie H-W, Cheng M-F, Tsai M-J, Chia Y-C, et al. Synergistic effect of natural compounds on the fatty acid-induced autophagy of activated hepatic stellate cells. J Nutr Biochem. 2014;25(9):903-13. doi:10.1016/j.jnutbio.2014.04.001.

32. Mannaerts I, Schroyen B, Verhulst S, Van Lommel L, Schuit F, Nyssen M, et al. Gene expression profiling of early hepatic stellate cell activation reveals a role for Igfbp3 in cell migration. PLoS One. 2013;8(12), e84071. doi:10.1371/journal.pone.0084071.

33. Mannaerts I, Leite SB, Verhulst S, Claerhout S, Eysackers N, Thoen LFR et al. The Hippo pathway effector YAP controls mouse hepatic stellate cell activation. Journal of Hepatology. Epub ahead of publication. doi:http:// dx.doi.org/10.1016/j.jhep.2015.04.011.

34. Miano JM, Long X, Fujiwara K. Serum response factor: master regulator of the actin cytoskeleton and contractile apparatus. 2007;292(1):C70-C81. doi:10.1152/ajpcell.00386.2006.

35. Herrmann J, Haas U, Gressner AM, Weiskirchen R. TGF- $\beta$ up-regulates serum response factor in activated hepatic stellate cells. Biochim Biophys Acta. 2007;1772(11-12):1250-7. http://dx.doi.org/10.1016/j.bbadis.2007.10.006.

36. Botella LM, Sanz-rodriguez F, Komi Y, Fernandez-I A, Varela E, Garrido-martin $E M$, et al. TGF- $\beta$ regulates the expression of transcription factor KLF6 and its splice variants and promotes co-operative transactivation of common target genes through a Smad3-Sp1-KLF6 interaction. Biochem J. 2009;419(2):485-95. doi:10.1042/bj20081434.

37. Kojima S, Hayashi S, Shimokado K, Suzuki Y, Shimada J, Crippa MP, et al. Transcriptional activation of urokinase by the Krüppel-like factor Zf9/COPEB activates latent TGF- $\beta 1$ in vascular endothelial cells. Blood. 2000;95(4):1309-16.

38. Zhang W, Ou J, Inagaki Y, Greenwel P, Ramirez F. Synergistic cooperation between Sp1 and Smad3/Smad4 mediates transforming growth factor $\beta 1$ stimulation of a2(I)-collagen (COL1A2) transcription. J Biol Chem. 2000;275(50):39237-45. doi:10.1074/jbc.M003339200.

39. Lee M, Song SU, Ryu JK, Suh JK. Sp1-dependent regulation of the tissue inhibitor of metalloproteinases-1 promoter. J Cell Biochem. 2004;91(6):1260-8. doi:10.1002/jcb.20021.

40. Park J-H, Jo J-H, Kim K-H, Kim S-J, Lee W-R, Park K-K, et al. Antifibrotic effect through the regulation of transcription factor using ring type-Sp1 decoy oligodeoxynucleotide in carbon tetrachloride-induced liver fibrosis. J Gene Med. 2009;11(9):824-33. doi:10.1002/jgm.1355.

41. Chen H, Zhou Y, Chen KQ, An G, Ji SY, Chen QK. Anti-fibrotic effects via regulation of transcription factor Sp1 on hepatic stellate cells. Cell Physiol Biochem. 2012;29(1-2):51-60.

42. Tsukamoto H, She H, Hazra S, Cheng J, Miyahara T. Anti-adipogenic regulation underlies hepatic stellate cell transdifferentiation. J Gastroenterol Hepatol. 2006;21:S102-5. doi:10.1111/j.1440-1746.2006.04573.x.

43. Madsen L, Petersen RK, Kristiansen K. Regulation of adipocyte differentiation and function by polyunsaturated fatty acids. Biochim Biophys Acta. 2005;1740(2):266-86. http://dx.doi.org/10.1016/j.bbadis.2005.03.001.

44. Galli A, Crabb D, Price D, Ceni E, Salzano R, Surrenti C, et al. Peroxisome proliferator-activated receptor $\gamma$ transcriptional regulation is involved in platelet-derived growth factor-induced proliferation of human hepatic stellate cells. Hepatology. 2000;31(1):101-8. doi:10.1002/hep.510310117.

45. Miyahara T, Schrum L, Rippe R, Xiong S, Yee HF, Motomura K, et al. Peroxisome proliferator-activated receptors and hepatic stellate cell activation. J Biol Chem. 2000;275(46):35715-22. doi:10.1074/jbc.M006577200.

46. Marra F, Efsen E, Romanelli RG, Caligiuri A, Pastacaldi S, Batignani G, et al. Ligands of peroxisome proliferator-activated receptor $y$ modulate profibrogenic and proinflammatory actions in hepatic stellate cells. Gastroenterology. 2000;119(2):466-78. http://dx.doi.org/10.1053/ gast.2000.9365.

47. Mikkelsen TS, Xu Z, Zhang X, Wang L, Gimble JM, Lander ES, et al. Comparative epigenomic analysis of murine and human adipogenesis. Cell. 2010;143(1):156-69. doi:10.1016/j.cell.2010.09.006.

48. Wakabayashi K-i, Okamura M, Tsutsumi S, Nishikawa NS, Tanaka T, Sakakibara I, et al. The peroxisome proliferator-activated receptor $\gamma /$ retinoid $X$ receptor a heterodimer targets the histone modification enzyme PR-Set7/Setd8 gene and regulates adipogenesis through a positive feedback loop. Mol Cell Biol. 2009;29(13):3544-55. doi:10.1128/mcb.01856-08.

49. Salma N, Xiao H, Mueller E, Imbalzano AN. Temporal recruitment of transcription factors and SWI/SNF chromatin-remodeling enzymes during adipogenic induction of the peroxisome proliferator-activated receptor $\gamma$ nuclear hormone receptor. Mol Cell Biol. 2004;24(11):4651-63. doi:10.1128/ mcb.24.11.4651-4663.2004.
50. Siersbæk R, Nielsen R, John S, Sung M-H, Baek S, Loft A, et al. Extensive chromatin remodelling and establishment of transcription factor 'hotspots' during early adipogenesis. EMBO J. 2011;30(8):1459-72. doi:10.1038/ emboj.2011.65.

51. Eeckhoute J, Oger F, Staels B, Lefebvre P. Coordinated regulation of PPARy expression and activity through control of chromatin structure in adipogenesis and obesity. PPAR Res. 2012;2012:9. doi:10.1155/2012/164140.

52. Imamura M, Inoguchi T, Ikuyama S, Taniguchi S, Kobayashi K, Nakashima N, et al. ADRP stimulates lipid accumulation and lipid droplet formation in murine fibroblasts. Am J Physiol Endocrinol Metab. 2002;283(4):E775-83. doi:10.1152/ajpendo.00040.2002.

53. Lee TF, Mak KM, Rackovsky O, Lin Y-L, Kwong AJ, Loke JC, et al. Downregulation of hepatic stellate cell activation by retinol and palmitate mediated by adipose differentiation-related protein (ADRP). J Cell Physiol. 2010;223(3):648-57. doi:10.1002/jcp.22063.

54. Guimarães ELM, Empsen C, Geerts A, van Grunsven LA. Advanced glycation end products induce production of reactive oxygen species via the activation of NADPH oxidase in murine hepatic stellate cells. J Hepatol. 2010;52(3):389-97. http://dx.doi.org/10.1016/j.jhep.2009.12.007.

55. Najimi M, Khuu DN, Lysy PA, Jazouli N, Abarca J, Sempoux C, et al. Adult-derived human liver mesenchymal-like cells as a potential progenitor reservoir of hepatocytes? Cell Transplant. 2007;16(7):717-28.

56. Coll M, Taghdouini AE, Perea L, Mannaerts I, Vila-Casadesús M, Blaya D et al. Integrative miRNA and gene expression profiling analysis of human quiescent hepatic stellate cells. Sci Rep. 2015;5. doi:10.1038/srep11549.

57. Mannaerts I, Nuytten NR, Rogiers V, Vanderkerken K, van Grunsven LA, Geerts A. Chronic administration of valproic acid inhibits activation of mouse hepatic stellate cells in vitro and in vivo. Hepatology. 2010;51(2):603-14. doi:10.1002/ hep.23334.

58. Irizarry RA, Bolstad BM, Collin F, Cope LM, Hobbs B, Speed TP. Summaries of Affymetrix GeneChip probe level data. Nucleic Acids Res. 2003;31(4), e15. doi:10.1093/nar/gng015.

59. Berardis S, Lombard C, Evraerts J, El Taghdouini A, Rosseels V, Sancho-Bru P, et al. Gene Expression Profiling and Secretome Analysis Differentiate Adult-Derived Human Liver Stem/Progenitor Cells and Human Hepatic Stellate Cells. PLOS ONE. 2014;9(1). doi:10.1371/journal.pone.0086137.

\section{Submit your next manuscript to BioMed Central and take full advantage of:}

- Convenient online submission

- Thorough peer review

- No space constraints or color figure charges

- Immediate publication on acceptance

- Inclusion in PubMed, CAS, Scopus and Google Scholar

- Research which is freely available for redistribution 